VOX PATRUM $31(2011)$ t. 56

Piotr KOCHANEK

(Lublin, KUL)

\title{
KONSTANTINOPEL IN DEN WELTKARTEN DES BEATUS VON LIÉBANA*
}

Beatus von Liébana (um 730-798) war Benediktinermönch, der im Kloster San Martin de Turieno im Tal von Liébana (Königreich Asturien im Norden Spaniens) lebte. Dieses Kloster trägt heute den Namen Santo Toribio de Liébana. ${ }^{1}$ Beatus ist bekannt vor allem durch einen aus zwölf Büchern bestehenden Apokalypsenkommentar. Eine erste Fassung dieses Kommentars wurde um 776 beendet und dann bis 786 neu redigiert ${ }^{2}$. Der Text des besagten Kommentars ist in 34 Handschriften und Fragmenten erhalten. Die Weltkarten befinden sich in 14 Handschriften, die zwischen dem 10. und dem 14. Jahrhundert entstanden sind ${ }^{3}$.

\footnotetext{
" Bei dem hier vorliegenden Artikel handelt es sich um eine erweiterte Version eines Kurzreferats, das auf dem 22. Internationalen Kongress für Byzantinische Studien, welches vom 22. bis 27. August 2011 in Sofia stattfand, gehalten wurde. Die Zusammenfassung des hier erwähnten Kurzreferats wurde in den Materialien zum Kongresses veröffentlicht, vgl. P. Kochanek, Konstantinopel in den Weltkarten des Beatus von Liébana, in: Proceedings of the 22nd International Congress of Byzantine Studies (Sofia, 22-27 August 2011), vol. II: Abstracts of Round Table Communications, ed. I. Iliev E. Kostova - V. Angelov, Bulgarian Historical Heritage Foundation, Sofia 2011, 275-276.

${ }^{1}$ Vgl. M. Férotin, Béatus, DB I 1531-1532; C. Verschaffel, Beatus de Liébana, DThC II $517-$ 518; J. Pérez de Urbel, Béat, DHGE VII 89-90; N. Crostarosa Scipioni, Beato di Liébana, ECat II 1108-1109; M. Salsano, Beato de Liebana, BS II 987-988; M.J. Daly, Beatus of Liébana, in: New Catholic Encyclopedia, II, San Francisco - Toronto 1967, 198; J.M. Szymusiak, Beatus z Liébana, EK II 163; J. Prelog - P.K. Klein, Beatus v. Liébana, in: Lexikon des Mittelalters, I, München Zürich 1980, 1746-1747; P.K. Klein, Beatus v. Liébana, LThK II 109.

2 Der besagte Kommentar wurde schon dreimal herausgegeben: 1. Sancti Beati, Presbyteri Hispani Liebanensis, in Apocalypsin, ac plurimas utriusque Foederis commentaria, ex veteribus, nonnullisque desideratis paginas Patribus, mille retro annis collecta, nunc primum edita, opera et studio R.P. Doct. H. Florez, Matriti 1770; 2. Beati in Apocalipsin libri duodecim, ed. H.A. Sanders, Rome 1930; 3. Sancti Beati a Liebana Commentarius in Apocalypsin, rec. E. Romero-Pose, vol. 1-2, Scriptores Graeci et Latini, Romae 1985; vgl. P. Kochanek, „Rozesłanie Apostołów” na mapie Beatusa z Burgo de Osma, in: Fructus Spiritus est Caritas, Księga Jubileuszowa ofiarowana Księdzu Profesorowi Franciszkowi Draczkowskiemu z okazji siedemdziesiatej rocznicy urodzin, czterdziestolecia święceń kapłańskich i trzydziestopięciolecia pracy naukowej, red. M. Wysocki, Lublin 2011, 707-708 und Anm. 19.

${ }^{3}$ Liste der in 14 Handschriften des Apokalypsenkommentars erhaltenen Beatuskarten: 1. Die Karte des Maius-Beatus = The Morgan Beatus (New York, Pierpont Morgan Library, Ms. 644, fol. 33ㄴ-34r). Entstehungszeit: 940-945. Größe: 530 x 380 mm. Format: rechteckig; 2. Die Karte des Valladolid-
} 
Es gibt noch zwei andere Beatuskarte: eine kaum beachtete Weltkarte, die sich außerhalb des Apokalypsenkommentars des Beatus befindet, d.h. die sogenannte

Beatus (Valladolid, Biblioteca de la Universidad, Ms. 433, fol. 36 $6^{\mathrm{v}}-37^{\mathrm{r}}$ ). Entstehungszeit: 970. Größe: 447 x 310 mm. Format: rechteckig, mit abgerundeten Ecken; 3. Die Karte des Gerona-Beatus (Gerona,

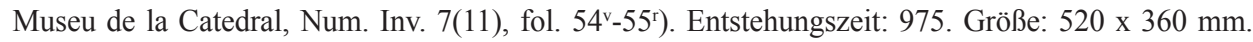
Format: rechteckig, mit abgerundeten Ecken; 4. Die Karte des Urgell-Beatus (Museu Diocesà de Seu d'Urgell, (Codex Urgellensis), Num. Inv. 501, fol. VIv-VII'). Entstehungszeit: zwischen 975 und 1000. Handschriftgröße: 402 x 265 mm. Format: rechteckig; 5. Die Karte des Facundus-Beatus (Madrid, Biblioteca Nacional, Ms. Vitrina 14-2, fol. 63ㄴ-64r). Entstehungszeit: 1047. Größe: 470 x 310 mm. Format: rechteckig, mit abgerundeten Ecken; 6. Die Karte des Saint-Sever-Beatus (Paris, Bibliothèque Nationale, Ms. Lat. 8878, fol. 45bisv-45ter ${ }^{r}$ ). Entstehungszeit: 1050-1075. Größe: 570 x 370 mm. Format: längsoval; 7. Die Karte des Osma-Beatus (Burgo de Osma, Archivo de la Catedral, Cod. 1, fol. 34ㄴ -35 ). Entstehungszeit: 1086. Größe: 380 x 300 mm. Format: oval; 8. Die Karte des Silos-Beatus (London, British Library, Add. Ms. 11695, fol. 39v-40r). Entstehungszeit: 1109. Größe: 488 x 375 mm. Format: rechteckig, mit abgerundeten Ecken; 9. Die Karte des Turin-Beatus (Turin, Biblioteca Nazionale Universitaria, Sfn. I.II.1, fol. $45^{\mathrm{v}}-46^{\mathrm{r}}$ ). Entstehungszeit: 1100-1125. Folioseite: $360 \times 275$ mm. Format: kreisrund; 10. Die Karte des Manchester-Beatus = The Rylands Beatus (Manchester, John Rylands University Library, Ms. Lat. 8, fol. 43v-44r). Entstehungszeit: ca. 1175 (bzw. Ende des 12.-Anf. des 13. Jh.). Handschriftgröße: 454 x 326 mm. Format: oval; 11. Die Karte des LorvãoBeatus (Lissabon, Arquivo Nacional da Torre do Tombo, Cod. 160 [alte Signatur]/Ms. CXIII/247 [neue Signatur], fol. 34bis ${ }^{v}$ ). Entstehungszeit: vor 1189. Folioseite: 340 x 230 mm. Format: kreisrund; 12. Die Karte des Navarra-Beatus (Paris, Bibliothèque Nationale, Nouv. acq. lat. 1366, fol. $24^{\mathrm{v}}-25^{\mathrm{r}}$ ). Entstehungszeit: Ende des 12.-Anf. des 13. Jh. Größe: 400 x 320 mm. Format: oval; 13. Die Karte des Las Huelgas-Beatus (New York, Pierpont Morgan Library, Ms. 429, fol. 31 ${ }^{\mathrm{v}}-32^{\mathrm{r}}$ ). Entstehungszeit: 1220. Größe: 560 x 380 mm. Format: oval; 14. Die Karte des Arroyo-Beatus (Paris, Bibliothèque Nationale, Nouv. acq. lat. 2290, fol. 13-14'). Entstehungszeit: 1200-1250. Folioseite: 457 x 300 mm. Format: kreisrund. Vgl. Literatur: B. Englisch, Ordo orbis terrae. Die Weltsicht in den Mappae mundi des frühen und hohen Mittelalters, Orbis Mediaevalis 3, Berlin 2002, 572-583 und 592-631; K. Miller, Mappaemundi. Die ältesten Weltkarten, 1. Heft: Die Weltkarte des Beatus, Stuttgart 1895, passim; M. Destombes, Mappemondes A.D. 1200-1500. Catalogue préparé par la Commission des Cartes Anciennes de l'Union Géographique Internationale, Monumenta Cartographica Vetustioris Aevi 1, Amsterdam 1964, 40-42 (Section 17) und 79-84 (Section 35); J. Strzelczyk, Gerwazy z Tilbury, in: Studium z dziejów uczoności w średniowieczu, Monografie z Dziejów Nauki i Techniki 66, Wrocław 1970, 208-212; A.D. von den Brincken, Fines Terrae. Die Enden der Erde und der vierte Kontinent auf mittelalterlichen Weltkarten, MGH Schriften 36, Hannover 1992, 56-58; J. Williams, The Illustrated Beatus: A Corpus of the Illustrations of the Commentary on the Apocalypse, vol. 1: Introduction, London 1994, 181(3); Ders., Isidore, Orosius and the Beatus Map, „Imago Mundi” 49 (1997) 10; I. Baumgärtner, Die Welt im kartographischen Blick. Zur Veränderbarkeit mittelalterlicher Weltkarten am Beispiel der Beatustradition vom 10. bis 13. Jahrhundert, in: Der weite Blick des Historikers: Einsichten in Kultur-, Landes- und Stadtgeschichte. Peter Johanek zum 65. Geburtstag, hrsg. W. Ehbrecht, Köln 2002, 527-549; Dies., Visualisierte Weltenräume. Tradition und Innovation in den Weltkarte der Beatustradition des 10. bis 13. Jahrhunderts, in: Tradition, Innovation, Invention. Fortschrittsverweigerung und Fortschrittsbewusstsein im Mittelalter, hrsg. H.J. Schmidt, Scrinium Friburgense 18, Berlin 2005, 263-273; Dies., Graphische Gestalt und Signifikanz. Europa in den Weltkarten des Beatus von Liébana und des Ranulf Higden, in: Europa im Weltbild des Mittelalters. Kartographische Konzepte, hrsg. I. Baumgärtner - H. Kugler, Orbis Mediaevalis 10, Berlin 2008, 83101; Kochanek, „Rozeslanie Apostołów”, S. 709-710, Anm. 24. 
Beatuskarte aus Oña ${ }^{4}$ und eine romanische Wandkarte in San Pedro de Rochas 5 . Heute sind also insgesamt 16 Beatuskarten bekannt.

Die Stadtvignetten Konstantinopels befinden sich auf vier Beatuskarten. Es handelt sich hier um: a) die Beatuskarte aus Saint-Sever (Paris, Bibliothèque Nationale, Ms. Lat. 8878, fol. 45bis ${ }^{\mathrm{v}}-45$ ter $^{\mathrm{r}}$ ), die in der südfranzösischen Abtei Saint-Sever-sur-l'Adour zwischen 1050 und 1075 entstanden sein dürfte, b) die Beatuskarte von Osma (Burgo de Osma, Archivo de la Catedral, Cod. 1, fol. $34^{\mathrm{v}}-35^{\mathrm{r}}$ ), datiert auf 1086, die im Cluniazenserkloster Sahagún im Bistum León gefertigt wurde, $\mathrm{c}$ ) die Beatuskarte von Navarra (Paris, Bibliothèque Nationale, Nouv. acq. lat. 1366, fol. $24^{\mathrm{v}}-25^{\mathrm{r}}$.) entstanden vermutlich in Navarra gegen Ende des 12. oder Anfang des 13. Jahrhunderts, und d) die Karte des Arroyo-Beatus (Paris, Bibliothèque Nationale, Nouv. acq. lat. 2290, fol. 13v$14^{\mathrm{r}}$ ), die in der Gegend um Burgos, wahrscheinlich in San Pedro de Cardeña, zwischen 1200 und 1250 gefertigt wurde ${ }^{6}$.

Die Randverzierungen von Konstantinopel auf den Beatuskarten aus Liébana werden hier folgendermaßen besprochen: 1. in diachronischer Reihenfolge, 2. verglichen mit den Vignetten der anderen vier Metropolen des Frühchristentums, das heißt mit den Vignetten Roms, Jerusalems, Antiochias und Alexandrias, und das sowohl synchronisch als auch diachronisch, so wie es die zwei weiter unten folgenden Tafeln zeigen. Das Ziel dieser Analysen ist ein Versuch, die Botschaft (bzw. den Code) zu ermitteln, die die Verfasser dieser Karten in den Vignetten von Konstantinopel zum Ausdruck bringen wollten.

Der diachronische Vergleich der Vignetten von Konstantinopel auf den vier Beatuskarten zeigt ihre „Entwicklung” von den einfachen Schemas der Beatuskarte von Saint-Sever bis hin zu der ,komplexen” Vignette der Beatuskarte aus Arroyo. Konstantinopel wird auf den Beatuskarten aus SaintSever, von Osma und von Navarra von einer Vignette repräsentiert, die ,keinen Sakralbau" darstellt.

\footnotetext{
${ }^{4}$ Mainland, Biblioteca Ambrosiana, Ms. F. 105 sup., fol. 71ํ- 72 ${ }^{\mathrm{r}}$. Entstehungszeit: Ende des 12. Jh. Format: oval; vgl. L. Vázquez de Parga, Un mapa desconocido de la serie de los „Beatos”, in: Actas del Simposio para el estudio de los códices del „Comentario al Apocalipsis” del Beato de Liébana, t. 1, Madrid 1978, 271-278; Kochanek, „Rozeslanie Apostołów”, S. 709-710, Anm. 24 und S. 740-741.

${ }^{5}$ Entstehungszeit: zwischen 1175 und 1200; vgl. J.M. García Iglesias, El mapa de los Beatos en la pintura mural románica de San Pedro de Rocas (Orense), „Archivos Leonenses” 69 (1981) 73 87; S. Moralejo, Mapa de la diáspora apostólica en San Pedro de Rocas: Notas para su interpretación y filiación en la tradición cartográfica de los „Beatos”, „Compostellanum” 31 (1986) 315-340; Kochanek, „Rozeslanie Apostołów”, S. 711 und 735-738.

${ }^{6}$ Die Entstehungszeit der Beatuskarten nach: Englisch, Ordo orbis terrae, S. 384 (die Karte des Navarra-Beatus), S. 617 (die Karte des Arroyo-Beatus), S. 625 (die Karte des Osma-Beatus), S. 629 (die Karte des Saint-Sever-Beatus). Vgl. auch Anm. 3
} 
Im Falle der Beatuskarte aus Saint-Sever erinnert uns dieses ,nicht sakrale Bauwerk" an eine Art Eingang zu einem Palast, obwohl man es auch als ein großes, aus drei Arkaden bestehendes Stadttor identifizieren könnte. Und diese Interpretation scheint auch die zutreffende Auslegung zu sein. Dieses Stadttor ähnelt mit seinem Aussehen der berühmten Porta Aurea in den Theodosianischen Mauern ${ }^{7}$. Es scheint, das dieses Motiv vom Illustrator der Beatuskarte von Osma übernommen wurde, der das Stadttor mit vier Andreaskreuzen ausschmückte. Eine Erklärung dafür wäre, dass der Körper dieses Apostels in der Konstantinopler Apostelkirche ruht. Die gleiche Vignette dieser Stadt in Form eines Tores wurde auch vom Illuminator der Beatuskarte von Navarra verwendet. Die zwei letzten Karten haben einerseits die Konstruktion des berühmten Tores vereinfacht und andererseits deutlich sichtbare Zinnen hinzugefügt. Das Tor war im Alten Testament, ähnlich wie die Mauer, ein Synonym für die Unabhängigkeit einer Stadt ${ }^{8}$. Konstantinopel wird hier als eine stark befestigte Stadt gezeigt. Diese Vignetten stehen jedoch in keinem Zusammenhang mit Konstantinopel im Sinne einer der Hauptmetropolen des Christentums. Diesen Umstand kann man dadurch erklären, dass alle drei oben genannten Karten (vielleicht ist hier die Beatuskarte aus Saint-Sever eine Ausnahme) nach 1054 entstanden, also nach dem Großen Schisma zwischen der Ost-Kirche Konstantinopels und der römischen West-Kirche. Die drei oben erwähnten Beatuskarten sprachen Konstantinopel die „Heiligkeit eines Ortes” ab, indem sie diese Vignetten verwendeten, damit zugleich auch das Recht auf den Rang

\footnotetext{
${ }^{7}$ Vgl. A.D. von den Brincken, Die Ausbildung konventioneller Zeichen und Farbgebungen in der Universalkartographie des Mittelalters, „Archiv für Diplomatik, Schriftgeschichte, Siegel- und Wappenkunde", 16 (1970) 345-346 (= Dies., Studien zur Universalkartographie des Mittelalters, hrsg. Th. Szabó, Veröffentlichungen des Max-Planck-Instituts für Geschichte 229, Göttingen 2008, 133: „Beatus verwendet drei- bis sechszinnige Bauten”; P. Arnaud, Les villes des cartographes. Vignettes urbaines et réseaux urbaines dans les mappemondes de l'Occident médiéval, „Mélanges de l'École Française de Rome. Moyen Âge - Temps Modernes” 96 (1984) 549: ,„...] la carte des manuscrits de Beatus [...] de Sainte Sever [...] représente la ville sous la forme d'une corniche à merlons supportée par des arcatures sur colonnes qui, [...], renvoient à des éléments réels d'architecture monumentale dont l'assemblage relève de la pure fantaisie"; Baumgärtner, Die Welt im kartographischen Blick, S. 540: „[...] individuellen Stadtvignetten [...]”; Dies., Visualisierte Weltenräume, S. 246: ,[...] individuellen Stadtvignetten [...]”.

${ }^{8}$ Vgl. M. Salamon, Rozwój idei Rzymu-Konstantynopola od IV do pierwszej połowy VI wie$k u$ (Zsfass.: The development of Rome-Constantinople from IV to the first half of the VI century), Prace Naukowe Uniwersytetu Śląskiego 80, Katowice 1975, 27; A. Buchalik, Tło w formie „bram i murów miast” na sarkofagach wczesnochrześcijańskich (Zsfass.: „City-gate” setting on Early Christian sarcophagi), VoxP 11-12 (1991-1992) Heft 20-23, S. 30. Was das Tor in der Bibel betrifft,

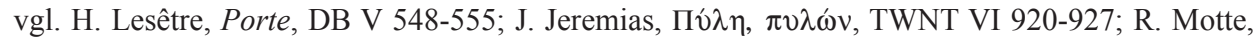
Porte, DB Supl VIII 126-136; Brama [Tor], in: F. Rienecker - G. Maier, Leksykon biblijny [Lexikon zur Bibel], übers. D. Irmińska, Warszawa 1994, 102-103; E. Otto, שֵׁِ ša ar, TWAT VIII 358-403 (Bibliographie, Sp. 358-364); Brama [Tor], in: Stownik symboliki biblijnej. Obrazy, symbole, motywy, metafory, figury stylistyczne i gatunki literackie w Piśmie Świętym [Dictionary of Biblical Imagery], hrsg. L. Ryken - J.C. Wilhoit, übers. Z. Kościuk, Warszawa 1998, 71-72.
} 
einer christlichen Metropole ${ }^{9}$. Somit kann man hier von einer bestimmten Tradition sprechen, Konstantinopel auf den drei oben genannten Vignetten der Beatuskarten aus Liébana ,nicht als eine sakrale” Stadt darzustellen.

Um eine ganz andere Vignette von Konstantinopel handelt es sich auf der Beatuskarte aus Arroyo. Sie erinnert an eine lateinische Kirche mit einem Turm ${ }^{10}$. Infolge dessen bricht die Vignette von Konstantinopel auf der Beatuskarte aus Arroyo auf der einen Seite etwas mit der zuvor beschriebenen Tradition, hat aber andererseits keinerlei Bezug zu der Architektur der Metropole am Bosporus. Das Anderssein dieser Vignette kann jedoch in einem bestimmten Maße die Entstehungszeit der Karte des Arroyo-Beatus erklären, das heißt der Zeitraum zwischen 1200 und 1250. Während des 4. Kreuzzuges (1202-1204) wurde Konstantinopel erobert und danach haben dort die Kreuzfahrer das Lateinische Kaiserreich errichtet. Dieses Reich existierte von 1204 bis 1261. In dem der Verfasser dieser Karte die Vignette in Form eines Schemas der lateinischen Kirche zeichnet, verkündet er irgendwie die Rückkehr Konstantinopels zur kirchlichen Einheit mit der West-Kirche. Die Hervorhebung des theologischpolitischen Aspekts ist klar, wenn man berücksichtigt, dass alle diese Karten in klösterlichen Skriptorien des Westens entstanden.

Demzufolge kann man in der Darstellung Konstantinopels auf den vier oben erwähnten Vignetten einen eindeutigen logischen Zusammenhang zwischen der Geografie, der Geschichte und der Theologie sehen.

\section{II.}

Die Beatuskarte aus Saint-Sever enthält die Vignetten aller fünf großen Metropolen des Christentums, das heißt Konstantinopel, Rom, Jerusalem, Antiochia und Alexandria. Die Vignetten dieser Städte sind sehr schematisch. Sie stellen Konturen von Toren (Konstantinopel, Rom ${ }^{11}$ und Antiochia) oder Schemas von Mauern mit Zinnen dar, in denen sich Tore befinden (Jerusalem ${ }^{12}$ und Alexandria). Die Konturen aller dieser Vignetten wurden mit roten Linien

\footnotetext{
${ }^{9}$ Vgl. Arnaud, Les villes des cartographes, S. 580-581.

${ }^{10}$ Vgl. Baumgärtner, Die Welt im kartographischen Blick, S. 539: „[...] individuell gestalteten Stadtsymbole"; Dies., Visualisierte Weltenräume, S. 245: „[...] individuell gestalteten Stadtsymbole [...]”; S. Sáenz-López Pérez, La Reconquista cartográfica: el Islam peninsular en la cartografía medieval hispana, „Treballs de la Societat Catalana de Geografia” 61-62 (2006) 289: „Desde el punto de vista artístico es interesante destacar las ciudades del mapa de San Andrés de Arroyo [...], donde se ilustran siguiendo el modelo de castillete gótico de tres torres destacando en altura la central [...]".

${ }^{11}$ Vgl. C. Cid, Santiago el Mayor en el texto y en las miniaturas de los códices del «Beato», „Compostellanum” 10 (1965) 266(622): „Roma, otra construcción rectangular con tres puertas, tres salientes redondeados y dos pequeños triángulos en la parte alta, como estibización de una basílica de tres naves con torres, que respondía a la realidad de aquella época".

${ }^{12}$ Vgl. Ebd., S. 266(622): „Ierusalem, sin duda la ciudad, porque es una especie de lienzo de muralla almenada con cinco puertas".
} 
gezeichnet (vgl. Tafel 1). Diese Konturen sind innen, mit der Ausnahme von Jerusalem, gelb ${ }^{13}$ ausgefüllt. Jerusalem besitzt im Gegensatz dazu grosso modo blau ausgefüllte Konturen. Darüber hinaus besitzt jede Kontur der Vignette zwei (Rom, Jerusalem, Antiochia und Alexandria) oder drei (Konstantinopel) Querstreifen ${ }^{14}$, die gleichfalls blau ausgefüllt sind, nur im Falle Jerusalems ist es Gelb. Der Farbton Blau, der diese Querstreifen ausfüllt, ist der gleiche wie das Blau, das die gesamte Kontur Jerusalems ausfüllt. Ferner durchfließt die Vignette von Rom der mit der gleichen Farbe markierte Fluss Tiber. Ein helles Grün füllt im Gegensatz dazu die Fenster und die Türen in den Konturen Konstantinopels, Roms und Antiochias aus. Man kann vielleicht in diesem Fall von einem bestimmten Farbcode sprechen ${ }^{15}$. Im weiteren erinnern die Vignetten der Beatuskarte aus Saint-Sever an ein bestimmtes System von Vignetten aus der Tabula Peutingeriana. Zweifelsohne ist dank der Anwendung eines Systems von sehr schematischen Vignetten Konstantinopel auf dieser Karte, trotz einer bestimmten ,Auszeichnung” Jerusalems, nicht als eine ,schlechtere/gebrandmarkte" Stadt dargestellt. Vielleicht verweist dies darauf, dass diese Karte vor dem Großen Schisma entstand und sich ihre Vignetten aus den römischen Wegekarten, wie z.B. die schon oben erwähnte Tabula Peutingeriana, oder aus Darstellungen der römischen Agrimensoren ableiteten ${ }^{16}$.

Die Beatuskarte von Osma besitzt keine „architektonische” Vignette Jerusalems. Sie besitzt dafür eine Vignette in Form des Kopfes des Apostels Jakobus (Jakobus der Jüngere) mit der Aufschrift: „Jerusalem. Jacobus frater Domini"17. Architektonische Motive treten im Gegensatz dazu auf den Vignetten der vier anderen Metropolen des Christentums auf. Auf der Vignette Konstantinopels ist es, wie schon weiter oben erwähnt wurde, ein Stadttor mit vier Andreaskreuzen, also ein Element des Verteidigungssystems. Ähnlich wurde Alexandria dargestellt: ein Fragment der Verteidigungsmauern sowie das Schema eines Leuchtturms ${ }^{18}$. Die Vignette Roms ist ein Porträt des Apostels Petrus, plat-

${ }^{13} \mathrm{Vgl}$. Von den Brincken, Die Ausbildung konventioneller Zeichen, Tafel zwischen S. 336 und S. 337 (= Dies., Studien zur Universalkartographie des Mittelalters, S. 124 [Tafel]); Arnaud, Les villes des cartographes, S. 562.

${ }^{14}$ Vgl. N. Duval, Représentations d'églises sur mosaïques, „Revue du Louvre” 22 (1972) 445: „[...] s'il est vrai qu'il faut considérer les bandes de couleur claire comme une traduction picturale de la sculpture architecturale".

${ }^{15} \mathrm{Vgl}$. Arnaud, Les villes des cartographes, S. 562, Anm. 83: „On a donc ici un authentique code de couleurs dans lequel le bleu est signe de sacré, et n'est nulle part aussi présent que dans Jérusalem. Le sacré, comme on le verra régulièrement est l'un des éléments essentiels de hiérarchisation des lieux cartographiques".

${ }^{16}$ Vgl. Von den Brincken, Die Ausbildung konventioneller Zeichen, S. 344 (= Dies., Studien zur Universalkartographie des Mittelalters, S. 132).

${ }^{17}$ Vgl. Miller, Mappaemundi. Die ältesten Weltkarten, 1. Heft, S. 35; vgl. auch Cid, Santiago el Mayor, S. 264(620): „Una modesta Jerusalén sin edificio, reducida a un basamento rectangular decorado con acantos y encima la cabeza de «Iacobus frater Domini»".

${ }^{18} \mathrm{Vgl}$. Arnaud, Les villes des cartographes, S. 552: „[Beatus de Burgo de Osma] a débaptisé 
ziert im Zentrum eines schematisch dargestellten Sakralbaus ${ }^{19}$. Ein ähnliches Schema eines Sakralbaus, aber ohne Porträt eines Apostels besitzt die Vignette Antiochias $^{20}$. Die Beatuskarte von Osma hebt also Rom und Jerusalem hervor. An die dritte Stelle in der Hierarchie platziert sie Antiochia. Im Gegensatz dazu werden Konstantinopel und Alexandria marginalisiert. Diese Marginalisierung Konstantinopels kann man damit erklären, dass die Karte im Cluniazenserkloster Sahagún nach dem Großen Schisma 1054 entstand. Dementsprechend ist die Beatuskarte von Osma ein Abbild einer bestimmten politischen Theologie, die von dem Cluniazensische Ordensverband vertreten wurde ${ }^{21}$.

Die Beatuskarte von Navarra, die vermutlich in Navarra gegen Ende des 12. oder Anfang des 13. Jahrhunderts gefertigt wurde, enthält eine große Anzahl von schönen Stadtvignetten. Bemerkenswert ist, dass diese Karte in sich „ein beachtliches Quantum der ungefähr 130 Toponyme, von denen immerhin 90 mit Namen von Provinzen, Regionen und Städten der bekannten Tabula Peutingeriana, [...] korrespondieren"'22, einschließt. Sie stellt Konstantinopel,

la ville pour accompagner la vignette de la légende «faro», qui n'est plus le nom propre «Pharos», mais le nom commun «phare» [...]".

${ }^{19}$ Vgl. Cid, Santiago el Mayor, S. 264(620): ,[...] Petrus [...], cuya cabeza enmarca un edficio con basamento, cuerpo principal con tres almenas y una torricidaexenta relacionada con el edificio por un breve enlace indefinible"; Ebd., S. 265(621): ,[...] sólo se representan dos iglesias con retrato, San Pedro y Santiago, ambas del mismo modelo, pero mucho mayor y más rica la segunda"; Arnaud, Les villes des cartographes, S. 559: „[...], le portrait du saint apparaît au centre d'une figure empruntée à l'architecture religieuse"; H. García-Aráez, Los mapamundis de los Beatos. Origen y características principales, „Miscelánea Medieval Murciana”, 18 (1993-1994) 72: „En la representación de algunas ciudades mediante aparatosos castillos, el [Beato de Osma] llega a presentar a Santiago de Compostela con castillo aún mayor que el de la propria Roma"; S. Moralejo, Las Islas del Sole. Sobre el mapamundi del Beato del Burgo de Osma (1086), in: A Imagem do mundo na Idade Média, Actas do Colóquio Internacional, organizadas por H. Codinho, com a colaboração de A.P. Morais e J.A. Frazão, Diálogo: Série Compilação, Lisboa 1992, 48 (= Ders., El mundo y el tiempo en el mapa del Beato de Osma, in: Patrimonio artístico de Galicia y otros estudios. Homenaje an Prof. Dr. Serafin Moralejo Álvarez, dir. Á.M. Franco Mata, 2. Bd, Santiago de Compostela 2004, S. 244): ,[...] a san Pedro en Roma se les concede el honor de enmarcar sus imágenes en la correspondiente basílica martirial [...]”;Baumgärtner, Die Welt im kartographischen Blick, S. 542 (= Dies., Visualisierte Weltenräume, S. 249): „Rom und Santiago de Compostela werden durch die geschickte Integration der Apostelköpfe von Petrus bzw. Jacobus in die Kirchengebäude demonstrativ akzentuiert”; J. Carracedo Fraga, Las „,sortes Apostolorum” en los „, Comentarios ai Apocalipsis” de Beato de Liébana y el mapamundi del 'Beato' de Burgo de Osma, „Euphrosyne” 38 (2010) 188: „Pedro y Santiago reciben un tratamiento distinguido; los dos están representados en el interior de su basílica de culto, con el objetivo claro de destacar por igual el carácter apostólico de ambos sedes".

${ }^{20}$ Vgl. I. Baumgärtner, Die Wahrnehmung Jerusalems auf mittelalterlichen Weltkarten, in: Jerusalem im Hoch- und Spätmittelalter. Konflikte und Konfliktbewältigung - Vorstellungen und Vergegenwärtigung, hrsg. D. Bauer - K. Herbers - N. Jaspert, Frankfurt am Main 2001, S. 286: ,[...] Antiochia und Tyrus als wehrhaftes Kirchengebäude bzw. befestigter Turm ausgearbeitet sind".

${ }^{21}$ Vgl. Moralejo, Las Islas del Sole, S. 43: ,[...] el mapa oxomense. Fruto de una geografía inseparable de la historia [...]".

${ }^{22}$ Baumgärtner, Die Welt im kartographischen Blick, S. 546; Dies., Visualisierte Weltenräume, S. 51. 
ähnlich wie die zwei oben besprochenen Karten, in Form eines Tores, also in Form eines nicht sakralen, sprich weltlichen Verteidigungsgebäudes. Fast genauso wurde Alexandria dargestellt: Fragmente von Verteidigungsmauern sowie ein Schema eines Leuchtturms ${ }^{23}$, was mit der Beatuskarte von Osma korrespondiert. Hingegen besitzen Rom, Jerusalem und Antiochia architektonische Vignetten, die schwer zu interpretieren sind. Carlos Cid scheint die Vignetten als Schemata von Kirchen zu interpretieren ${ }^{24}$. Im Zusammenhang mit den Vignetten Jerusalems und Antiochias sollte man anmerken, dass sich diese Städte in den folgenden Zeiträumen in den Händen der Kreuzfahrer befanden: Jerusalem - 1099-1187 und 1229-1244; Antiochia - 1098-1268. Aus diesem Grund können diese Vignetten eine sakrale Architektur enthalten, obwohl die Vignette Jerusalems doch eher ein Verteidigungstor darzustellen scheint. Eine solche Interpretation dieser Vignette verweist auf eine Stadt, die im Verständnis des Kartenverfassers ihren ,sakralen” Charakter verlor, nachdem sie (1187-1229 oder definitiv nach 1244) in die Hände der Muselmanen überging. Antiochia hat gleichfalls Charakterzüge einer Festung - gewaltige Türme auf beiden Seiten des „Tores”. Und in der Mitte der Stadt sieht man drei hohe Türme mit Kuppeln, wobei die mittlere Kuppel die Höchste ist. Es können Türme von christlichen Kirchen sein, aber es können auch Türme von Minaretten sein, die in Antiochia nach 1268 gebaut wurden. Wenn man die letztere These annehmen würde, müsste man den Entstehungszeitpunkt der Beatuskarte von Navarra auf die Zeit nach 1268 legen.

Die Karte des Arroyo-Beatus entstand vermutlich, wie schon oben erwähnt wurde, zwischen 1200 und 1250. Eben in diesem Zeitraum war Konstantinopel die Hauptstadt des Lateinischen Kaiserreichs. Dieses Reich existierte, wie man weiß, zwischen 1204 und 1261. Es gab auch zwischen 1098 und 1268 das Fürstentum Antiochia. Schließlich befand sich Jerusalem in den Jahren 1229-1244 wieder in den Händen der Lateiner, obwohl im Grunde genommen ihre Situation im Nahen Osten immer kritischer wurde. Infolge dessen waren Antiochia und Jerusalem zu jener Zeit besonders der Gefahr ausgesetzt, von muselmanischen Armeen angegriffen zu werden. Und eben dieser Umstand scheint die Form der architektonischen Vignetten dieser Städte gut zu erklären. Man kann sie als Schemata sakraler Gebäude mit Elementen der Verteidigungsarchitektur betrachten. Auf der anderen Seite muss man sehen, dass fast alle Vignetten auf der Karte des Arroyo-Beatus ein ähnliches Schema besitzen wie Jerusalem und Antiochia. Das hatte vermutlich etwas mit der stürmischen Geschichte des Christentums in der ersten Hälfte des 13. Jahrhunderts zu tun: ständige Kämpfe der kleinen Staatsgebilden der Kreuzfahrer im Nahen

${ }^{23}$ Vgl. Moralejo, El mundo y el tiempo, S. 247: „El llamado Beato Navarro o Gascón retendrá sólo el faro de Alejandría, reconocible también, como torre almenade”.

${ }^{24}$ Vgl. Cid, Santiago el Mayor, S. 263(619): „Es (el mapa) muy rico de detalles, entre ellos grandes edificios que representan iglesias". 
Osten ums Überleben; ständige Kämpfe mit den Muselmanen in Spanien sowie der schockierende Angriff der Mongolen auf Europa im Jahre 1241. Die Mongolen erreichten damals die kroatische Adria. Nach der Schlacht vom Köse Dăg im Jahre 1243 wurden auch die Rum-Seldschuken in Kleinasien mongolische Vasallen. Die damalige Welt war ein Schlachtfeld. Das Christentum ging in die Defensive und eben dies verbildlicht die Verteidigungsarchitektur der Vignetten der Karte des Arroyo-Beatus. In diesem Zusammenhang ist es angebracht, die Suggestion von Sandra Sáenz-López Pérez anzuführen, dass das Modell für diese Vignetten ,castillete gótico de tres torres destacando en altura la central" war ${ }^{25}$. Für den Verfasser der Karte ist die Welt nicht, wie es einige wollen, eine Welt von monumentalen und reichen Bauwerken ${ }^{26}$, sondern eine Welt von starken Verteidigungsbauwerken. Gleichzeitig suggerieren diese Tatsachen einen Entstehungszeitraum für diese Karte auf die Jahre 12291244. Hat die Vignette Konstantinopels auch ein Schema einer solide gebauten lateinischen Kirche mit einem Turm (ähnlich wie die Vignetten: Abcustria, Aquileia, Ascalon, Babilonia, Beneabento, Gens, Geramontes, Germania und Irlanda) ${ }^{27}$, so erinnern die Vignetten Roms, Jerusalems und Antiochias an Kirchen mit einzelnen hohen Türmen, die von Verteidigungsmauern umgeben sind, und um genau zu sein, die von Mauerfragmenten mit einem Tor umgeben sind, das von zwei niedrigeren Türmen flankiert wird. Auf diese Art entstand ein Schema von drei Türmen, von denn sich der mittlere Turm durch seine Höhe hervorhob. Dabei unterscheidet sich der mittlere Turm Jerusalems und Antiochias von den Türmen Konstantinopels und Roms. Die mittleren, hohen Türme Jerusalems und Antiochias scheinen den berühmten, unter dem Namen die Giralda bekannten Turm als Vorbild gehabt zu haben. Es ist das Minarett der Moschee von Sevilla, die zwischen 1195 und 1197 gebaut wurde. Nach der christlichen Rückeroberung dieser Stadt im Jahre 1248 funktionierte die Moschee als Kirche. Dieses auf der Iberischen Halbinsel gut bekannte Bauwerk hat wahrscheinlich den Illustrator der Beatuskarte aus Arroya inspiriert. Auf jeden Fall fügte er im ,spanischen” Teil seiner Karte einige sehr große Vignetten mit einem hohen mittleren Turm ein (vgl. Abb. 1: Betica, Cesaraugusta, Seuilla, Toletum) und dieses gleiche Motiv wiederholte er auf den meisten Vignetten der von ihm illustrierten Karte. In diesem Zusammenhang lohnt es sich, an die These von Hermann Thiersch (1874-1939) aus dem Jahr 1909 zu erinnern, die davon ausgeht, dass die Architektur dieses Minaretts von dem berühmten Großen Leuchtturm von Alexandria inspiriert wurde und dass später viele Baumeister von mittelalterlichen Kirchtürmen dieses Modell übernahmen ${ }^{28}$.

${ }^{25} \mathrm{Vgl}$. Anm. 10.

${ }^{26}$ Vgl.. Cid, Santiago el Mayor, S. 267(623): ,edificios monumentales y ricos”.

${ }^{27}$ Die lateinischen geographischen Namen nach: Miller, Mappaemundi.Die ältesten Weltkarten, 1. Heft, S. 38-40.

${ }^{28} \mathrm{Vgl}$. H. Thiersch, Pharos. Antike Islam und Occident. Ein Beitrag zur Architekturgeschichte, Leipzig - Berlin 1909, 126-127; vgl. auch ebd., S. 192, Abb. 335: Campanile der Hauptkirche in Lecce; 
Der Illustrator der Karte musste also die antike Genese der Giralda in Sevilla nicht kennen, aber dadurch, dass er von dem Bau inspiriert wurde, führte er unbewusst eine Reminiszenz an den Leuchtturm aus Pharos ein, obwohl er weder Alexandria noch ihren Leuchtturm auf seiner Karte platzierte.

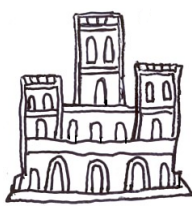

A.

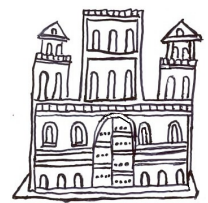

B.

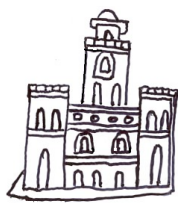

C.

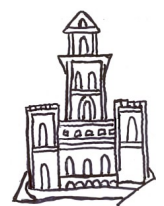

D.

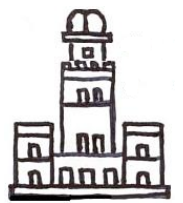

E.

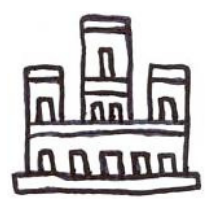

F.

Abb. 1: Beatuskarte aus Arroyo: A. Seuilia; B. Toletum; C. Betica; D. Cesaraugusta; E. Jhrlm (Jerusalem); F. Antiochia ${ }^{29}$

Die Position Konstantinopels auf dieser Karte wird hier aus dem historischen Kontext erschlossen. Der Entstehungszeitpunkt dieser Karte ist der Zeitraum einer bestimmten scheinbaren territorialen Renaissance des westlichen Christentums: Es besaß zu jener Zeit vier große Kirchenmetropolen: Antiochia, Jerusalem, Konstantinopel und Rom, und die spanische ,Reconquista" reichte bis nach Sevilla. Auf der anderen Seite wurde die damalige Welt von ,globalen" militärischen Konflikten erschüttert. Der Verfasser der Beatuskarte aus Arroyo war bemüht, diese Aspekte der damaligen Wirklichkeit darzustellen.

\section{III.}

Die Stadtvignetten Konstantinopels in den Weltkarten des Beatus von Liébana sind mit dem Zeitraum der jeweiligen Karte aufs Engste verbunden. Mit anderen Worten: Geografie, Geschichte und politische Theologie sind hier miteinander eng verflochten ${ }^{30}$. Die analysierten Vignetten der Beatuskarten illustrieren Veränderungen in der Herangehensweise an die Fragen der Rolle

S. 192, Abb. 336: Campanile in Soleto (Apulien); S. 195, Abb. 356: Campanile der Kathedrale von Gaëta; vgl. auch K. Deppmeyer, Der Leuchtturm von Pharos - ein spätes Weltwunder, „Frankfurter elektronische Rundschau zur Altertumskunde" 3 (2006) 4 [= http://www.fera-journal.eu (10.08.2011)]: „Eine Renaissance erlebte der Leuchtturm erst zu Beginn des letzten Jahrhunderts als ihn Hermann Thiersch mit einer umfassenden Monographie wieder ins Bewusstsein brachte. Die Arbeit ist auch heute noch für viele Aspekte hilfreich. So machte Thiersch auf die Vorbildwirkung des Pharos für die Minarettbaukunst aufmerksam, doch sind seine Ausführugen teilweise zu relativieren. m. E. ist die Aussage, dass der Pharos eine spezielle Nachwirkung sowohl bei gotischen Türmen als auch mittelalterlichen Glockentürmen zeitigte, abzulehnen. Doch ist die Gestalt früher ägyptischer und spanischer Minarette durchaus mit dem Pharos vergleichbar".

${ }^{29}$ Die lateinischen geographischen Namen nach: Miller, Mappaemundi. Die ältesten Weltkarten, 1. Heft, S. 38 und 40.

${ }^{30} \mathrm{Vgl}$. Anm. 21. 
und der Bedeutung Konstantinopels in den Augen der Lateiner im Zeitraum von 1050 bis 1250. Die Vignetten sind gewisse Schemata, die vor allem für die Menschen jener Zeit bestimmt waren und von ihnen verstanden wurden. Diese Vignetten bilden kein universales System ${ }^{31}$, daher versah jeder Kartenverfasser sie mit Beschriftungen, um Zweideutigkeiten bei der Identifizierung der jeweiligen Städte zu vermeiden ${ }^{32}$. Sie sind jedoch verständlich im Rahmen einer konkreten Karte, wenn man sie vor dem damaligen geopolitischen und theologischen Hintergrund analysiert. Die damalige Vignette Konstantinopels erscheint als ein in ihrer Art besonderes Abbild der Einstellung der Lateiner den Griechen gegenüber.

\section{CONSTANTINOPLE IN THE WORLD MAPS OF THE BEATUS OF LIÉBANA}

\section{(Summary)}

Vignettes of Constantinople are on four world maps of the Beatus of Liébana (c. 730798): 1) the Beatus map of Saint-Sever (Paris, Bibliothèque Nationale, Ms. Lat. 8878, fol. 45bis ${ }^{\mathrm{v}}-45 \operatorname{ter}^{\mathrm{r}}$ ), 2) the Beatus map of Osma (Burgo de Osma, Archivo de la Catedral, Cod. 1, fol. $\left.34^{\mathrm{v}}-35^{\mathrm{r}}\right), 3$ ) the Beatus map of Navarra (Paris, Bibliothèque Nationale, Nouv. acq. lat. 1366, fol. $24^{\mathrm{v}}-25^{\mathrm{r}}$.), and 4) the Beatus map of Arroyo (Paris, Bibliothèque Nationale, Nouv. acq. lat. 2290 , fol. $13^{\mathrm{v}}-14^{\mathrm{r}}$ ). These vignettes are with the period of origin of each map closely connected. In other words, geography, history and political theology are here intimately linked. The vignettes analyzed of the word maps of Beatus illustrate changes in the approach to the question of the role and significance of Constantinople in the eyes of the Latins in the period from 1050 to 1250 . These vignettes were certain schemes, that were designed especially for the people of that time and were understood by them. The vignettes were not a universal system, so every mapmaker adorned them with labels in order to avoid ambiguities in the identification of the respective cities. These vignettes were, however, understandable in the context of a specific map, when the map was analyzed against the then geopolitical and theological background. In consequence of this

${ }^{31}$ Vgl. Von den Brincken, Die Ausbildung konventioneller Zeichen, S. 325 (= Dies., Studien zur Universalkartographie des Mittelalters, S. 112): „Diese Zeichensprache ist jedoch in Antike und Mittelalter nicht ausgebildet; auch gibt es jeweils keine individuelle Zusammenstellung der Zeichen für die einzelnen Blätter"; vgl. auch ebd., S. 336.347 (= Dies., Studien zur Universalkartographie des Mittelalters, S. 122. 134); J.G. Deckers, Tradition und Adaptation. Bemerkungen zur Darstellung der christlichen Stadt, in: Actes du XI Congrès International d'Archéologie Chrètienne (Lyon Vienne - Grenoble - Aoste, 21-28 septembre 1986), vol. 2, Collection del'École Française de Rome 123. Studi di Antichità Cristiana 41, Città del Vaticano - Rome1989, 1286; K. Zalewska-Lorkiewicz, Ilustrowane „mappae mundi” jako obraz świata. Średniowiecze i poczatek okresu nowożytnego [Illustrierte mappae mundi als Weltbild. Mittelalter und Früher Neuzeit], Res Humanae. Studia 3, Warszawa 1997, 31.

${ }^{32} \mathrm{Vgl}$. Discussion [Prof. J. Engemann], in: Actes du XI Congrès International d'Archéologie Chrètienne, vol. 2, S. 1369. 
the vignettes of Constantinople on four world maps of the Beatus of Liébana appears as a particular image of the relations between the Latins and the Greeks.

\section{Tafel 1: Der originalen Stadtvignetten}

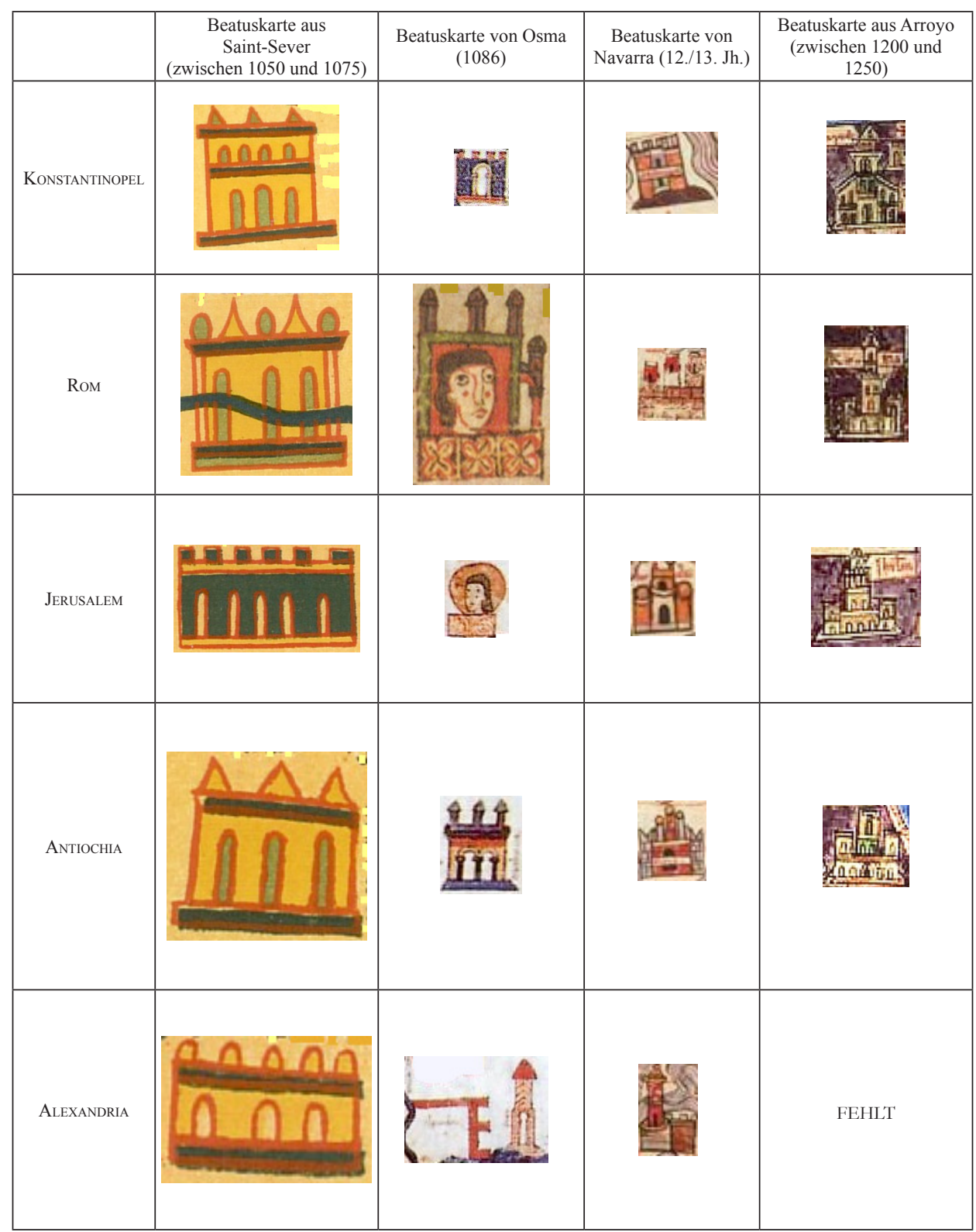


Tafel 2: Der Durchzeichnungen der Stadtvignetten

KonStantinope

${ }^{33}$ Die Stadtpiktogramme dieser Beatuskarte nach: Miller, Mappaemundi. Die ältesten Weltkarten, 1. Heft, S. 35 .

${ }^{34}$ Die Stadtvignetten Roms und Alexandrias nach: Ebd., S. 31.

${ }^{35}$ Die Stadtpiktogramme dieser Beatuskarte (mit Ausnahme Jerusalems) nach: Ebd., S. 39. 


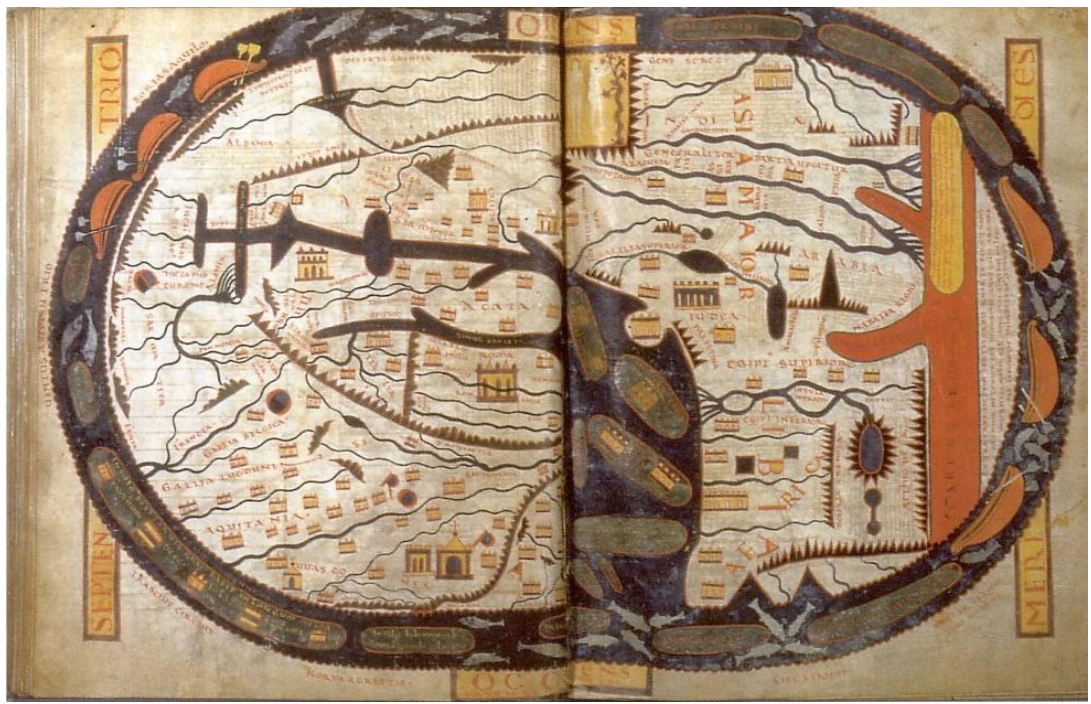

Abb. 2: Beatuskarte aus Saint-Sever (Paris, Bibliothèque Nationale, Ms. Lat. 8878, fol. 45bis ${ }^{\mathrm{v}}-45$ ter $^{\mathrm{r}}$ ); Entstehungszeit: zwischen 1050 und 1075 (nach: Englisch, Ordo orbis terrae, S. 365, Abb. 63)

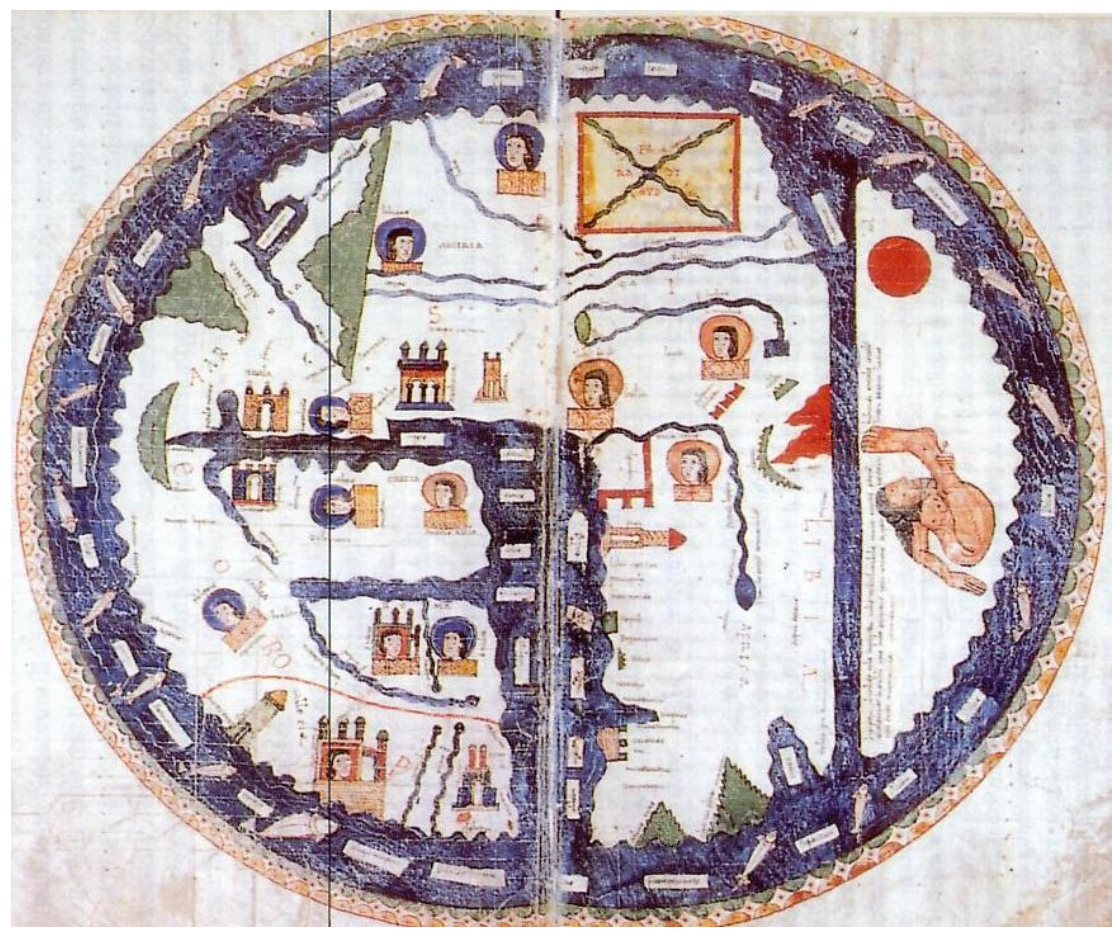

Abb. 3: Beatuskarte von Osma (Burgo de Osma, Archivo de la Catedral, Cod. 1, fol. 34v-35r); Entstehungszeit: 1086 (nach: Englisch, Ordo orbis terrae, S. 345, Abb. 61). 


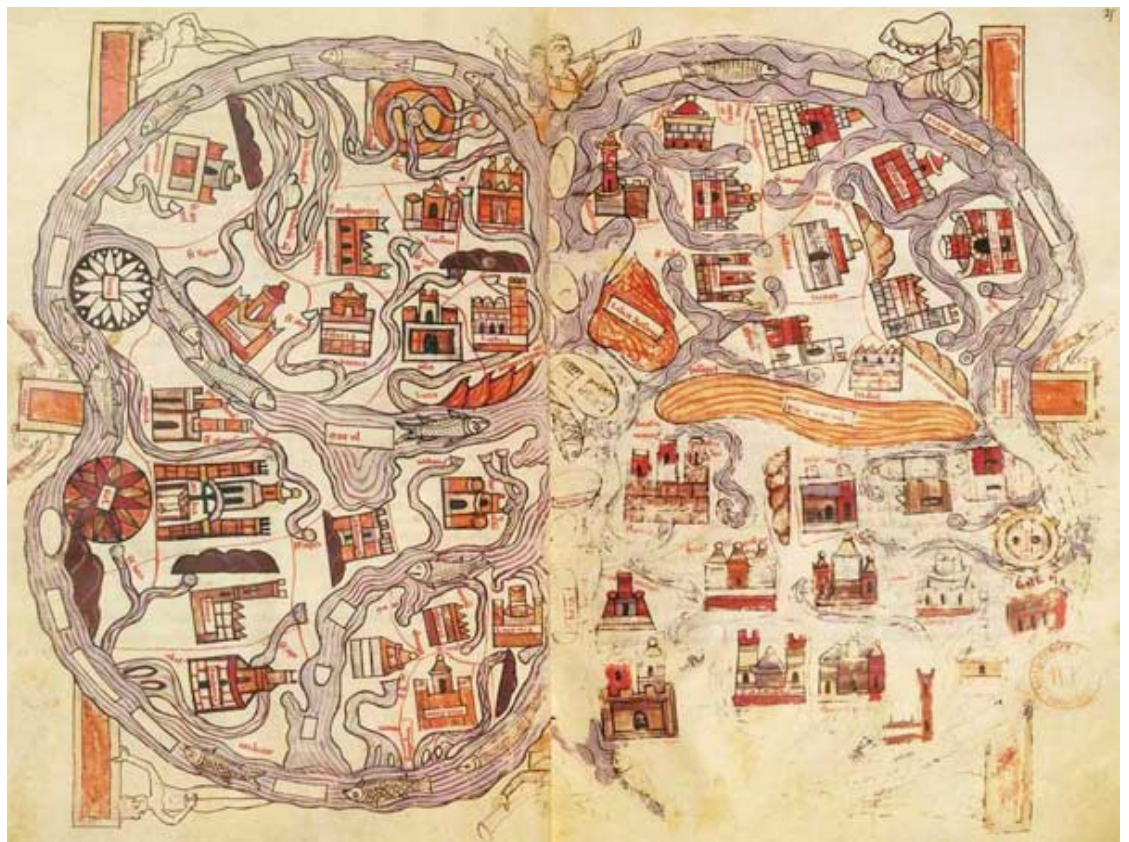

Abb. 4: Beatuskarte von Navarra (Paris, Bibliothèque Nationale, Ms. nouv. acq. Lat. 1366, fol. 24 ${ }^{\mathrm{v}}-25^{\mathrm{r}}$ ); Entstehungszeit: 12/13. Jh. (nach: www.codicesillustres.com/catalogue/navarra_beatus/index.html [2.08.2011])

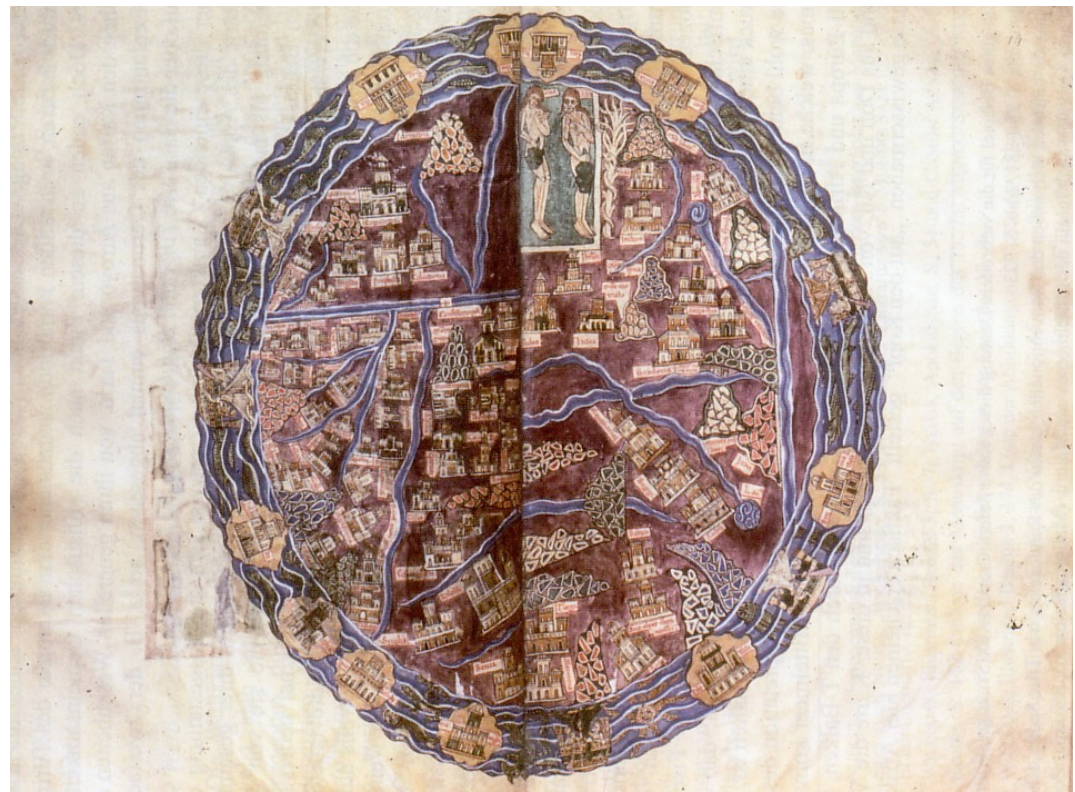

Abb. 5: Beatuskarte aus Arroyo (Paris, Bibliothèque Nationale, Ms. nouv. acq. lat. 2290, fol. 13는 $14^{\mathrm{r}}$ ); Entstehungszeit: zwischen 1200 und 1250 (nach: Englisch, Ordo orbis terrae, S. 326, Abb. 57). 
